of the precursor does not seem to be due to carbohydrate, for neuraminidase failed to alter its migration and it is unlikely that the reticulocyte lysate would contain the specific polysaccharide machinery for synthesizing. It therefore seems that light chains are made in the form of a slightly larger precursor, and that the vesicles of microsomes are required for converting this precursor to its final form. Reticulocyte lysates contain only free polysomes while ascites cell extracts probably contain some membraneous material thus allowing for the conversion of the precursor to light chains.

The authors further speculate that the short amino-acid sequence at the $\mathrm{N}$-terminus of the precursor may be the signal for membrane attachment of polysomes, an event which occurs after chain initiation has begun, and therefore may play a part in the secretory process. The precursors of mRNA and proteins will be the subject of much work in the next few years.-From a Correspondent.

\title{
Moon-making in Three Dimensions
}

ALmost every theory of the formation of the planetary system requires the existence of a "solar nebula" in the broadest sense of some diffuse material in the vicinity of the Sun at some early stage in its evolution. Most theories regard the material as having originally about the same relative abundances of the elements as in the Sun. They generally regard it as occupying about the region of the present system in the sense that the material had to be in this region for anything relevant to happen to it. Otherwise theories differ enormously in the picture of the solar nebula, treating it as a regular distribution of material having some simple motion of circulation about the Sun, or as forming a pattern of large vortices, or as highly turbulent material, or as part of a more extensive cloud with which it continues to exchange material and momentum, or as material controlled by a magnetic field, and so on.

In his article on the origin of the Moon on page 263 of this issue of Nature, Don L. Anderson uses the essentially simplest picture of the initial state of the nebula, but it is well to remember that even as a starting point it is only one of the array of possibilities just mentioned. On the adopted picture, cosmogonists discuss the distribution of temperature and pressure and thence infer the extent to which elements and compounds (metals, carbon, silicates, ices, and so on) condense out of the gaseous phase to form small solid grains, or to which gaseous components may be driven out of the nebula. Thus they derive for the relevant epoch a chemical composition depending primarily upon distance from the Sun. Each planet is then supposed to "accumulate" from the material of the nebula in an appropriate zone, the different compositions of the planets being supposed to depend primarily upon the differences between the zones, although time-dependences of some of the process may also have an effect.

Coming to satellites, theories differ greatly depending upon whether they emphasize similarities or differences between the planetary system and a satellite system. Again, theories of the origin of the Moon differ according to whether they emphasize its similarity to the other principal satellites regarding absolute dimensions, or its difference from them regarding dimensions relative to those of the associated planet (the ratio of the mass of the Earth to that of the Moon being almost ten times greater than for any other such pair). Anyhow, at present interest is mostly focused upon the Earth-Moon system simply because empirical knowledge about it is incomparably greater than about any like system. Also the Apollo missions have almost given the illusion that as much is now known about the Moon as about the Earth.

One obvious problem presented by the pair is that of their exceedingly different composition, which places the "accumulation" theory in a serious dilemma. For if Earth and Moon accumulated in the same zone, we should expect them to have similar compositions. On the other hand, if the Moon accumulated somewhere else in the system and was then captured by the Earth, it would be an example of a body whose composition is not characteristic of the zone where we now find it; but this almost contradicts the case for explaining the composition of bodies by a zoning of materials.

Anderson finds a way out of this dilemma effectively by going into an extra dimension. He points out that the calculation of the composition of the solar nebula would give a dependence upon distance from its median plane as well as distance from the Sun. Whereas the Earth may be supposed to have accumulated close to the plane, if the Moon formed by accumulation it would necessarily have drawn its material from a much larger range of distance on either side of the plane. Anderson obviously finds that the pursuit of this idea and its testing taxes all the manifold resources of the relevant chemistry and mineralogy, but he evidently draws encouragement from the results, so far as they go.

Anderson's ideas are in a general way similar to those of A. E. Ringwood (J. Geophys. Res., 75, 6453; 1970), which have been developed by other authors as well. Ringwood takes account of the time-sequence of various processes, as does Anderson, but Anderson's idea of also taking account of the variation of composition away from the median plane is apparently new in this context.

The fission theory (in any of its forms) and the capture theory of lunar origin have met with such severe criticisms that it has become a challenge to the "accumulation" theory to show that it can do better. Anderson's work and the like certainly shows that it meets with no obvious contradiction. But it then becomes so complicated that it is hard to see how any crucial test could be proposed within the frame of the work itself. Hopes of some decision seem to lie in two directions - first, the mechanics of the process: it requires to be shown that the accumulation process would actually operate and that it would be expected to produce one Moon with the correct mass, spin momentum, distance from the Earth and so on; second, comparative studies: it requires to be shown whether the processes proposed would account for the Galilean satellites of Jupiter, Titan (Saturn) and Triton (Neptune), all of which have mass and size very similar to those of the Moon.-W. H. McC. 